\title{
Anaplastic thyroid carcinoma: a nationwide cohort study on incidence, treatment and survival in the Netherlands over 3 decades
}

\author{
Mischa de Ridder ${ }^{\mathbb{1} 1}$, Els Nieveen van Dijkum², Anton Engelsman², Ellen Kapiteijn ${ }^{3}$, Heinz-Josef Klümpen and \\ Coen R N Rasch'
}

${ }^{1}$ Department of Radiation Oncology, Leiden University Medical Centre, Leiden, The Netherlands, ${ }^{2}$ Department of Surgery, Cancer Center Amsterdam, Amsterdam University Medical Center, University of Amsterdam, Amsterdam, The Netherlands, ${ }^{3}$ Department of Medical Oncology, Leiden University Medical Centre, Leiden, The Netherlands, and ${ }^{4}$ Department of Medical Oncology, Cancer Center Amsterdam, Amsterdam University Medical Center, University of Amsterdam, Amsterdam, The Netherlands

Correspondence should be addressed to $\mathrm{M}$ de Ridder

Email

M.de_Ridder.RT@lumc.nl

\section{Abstract}

Objective: To perform a nationwide population based study in ATC on incidence, treatment and survival.

Design: Retrospective cohort study.

Methods: All patients with primary ATC between 1989 and 2016 were identified in the Netherlands Cancer Registry (NCR). Of all these patients excerpts from the pathology reports from PALGA: Dutch Pathology registry were linked to the data of the NCR. Standardized incidences were calculated, survival was estimated using Kaplan-Meier method and univariable statistically significant factors were included in a multivariable regression model.

Results: In total, 812 patients were included. Mean standardized incidence rates were 0.18/100 000 (range $0.11-0.27 / 100000$ ) with a significant trend over the years with an estimated annual percentage change of $1.3 \%$ per year (95\% Cl 0.4-2.1\%). Median overall survival was 2.2 months, and estimated 1-year survival was $12 \%$. Patients without distant metastases at diagnosis had an estimated 1-year survival of 21.6\%. Prognostic factors for prolonged survival were double or triple therapy, age below 65 years, M0-status and absence of bilateral lymph node metastases. Conclusions: ATC is rare, but often lethal, form of thyroid cancer, with a median survival of 2 months and 1-year survival of approximately $10 \%$. The incidence is slightly rising in the Netherlands over the past 3 decades. There appears to be a subgroup of patients that survive longer, mainly those with relatively limited disease who underwent double or triple therapy. Further research is needed to define these patients more distinctively.

\section{Introduction}

Anaplastic thyroid carcinoma (ATC) is one of the most aggressive solid tumors. The prognosis of this tumor can be classified as very poor, with a median survival of 3-6 months (1), mainly due to diffuse distant metastases and/or rapid local growth compromising vital structures in the neck (2). Anaplastic thyroid carcinoma accounts for $50 \%$ of the thyroid-cancer related mortality, but due to its low incidence occurs in less than $2 \%$ of all thyroid cancers
$(1,3,4)$. Randomized trials on treatment options are lacking due to the rarity of the cancer type and the overall poor prognosis. The American Thyroid Association published the guideline on ATC, in 2012 (5), and the NCCN in 2015 (6), providing guidance for diagnosis, treatment, palliative care and monitoring. An interesting side evaluation of the guideline showed that the overall strength and quality of the evidence is poor, indicating, 
furthermore, that high-quality literature is lacking. Based on clinical practice and several single institutional studies $(7,8,9,10)$, there appears to be a subgroup of ATC patients with an better than expected survival. Aim of this study was to perform a nationwide population based study in ATC patients on incidence, treatment and survival, with the goal to confirm that there appears to be a subgroup of patients with a better prognosis and also explore the clinical characteristics of these patients.

\section{Patients and methods}

\section{Data sources}

All patients diagnosed with primary ATC between 1989 and 2016 in The Netherlands were identified in The Netherlands Cancer Registry (NCR) and included in the study.

The NCR is population-based and cancer cases are identified from pathology records received from the nationwide network and registry of histo- and cytopathology in the Netherlands (PALGA), as well as from the hospital discharge registry. The completeness of the NCR was estimated to be equal to at least 95\% (11). Following notification, trained tumor registration clerks abstract a minimum data set, including patient, tumor and treatment characteristics from hospital records.

Including all patients with ATC from the PALGA network formed a second dataset. The PALGA registry has a national coverage of pathology reports since 1991. It receives its input data automatically from all pathology laboratories. Patients are de-identified and excerpts of the pathology reports containing the age and sex of the patient, as well as the date, diagnosis and conclusion of the report are available for research questions from third parties. The dataset of the NCR was linked to PALGA data by a trusted third party. This was done in order to check eventual misclassified patients in the NKR dataset and to gain extra information on type of surgery performed, thereby creating an optimal nationwide database of ATC patients over 1989-2016.

\section{Definitions}

Patients were classified based on ICD-O-3 (12) code: topography C739 for thyroid and morphology 8012, $8020,8021,8022,8030,8031,8032,8033$ and 8035. In the PALGA database, the terms Thyroid (T96) and Anaplastic carcinoma (M80321) or Thyroid (T96) and Anaplastic
(M72005) and Carcinoma (M80103) were used. Also plain text searches with terms Anaplastic Thyroid Carcinoma were done.

TNM classification was done according to the version available at time of diagnosis. So, from 1989 to 1998 the 4th edition was used, 1999 to 2002 the 5th edition, 2003 to 2009 the 6th edition and from 2010 to 2016 the 7th edition.

Treatment was classified into groups: surgery only, surgery plus radiotherapy (RT), surgery plus concurrent chemoradiotherapy (cCRT), surgery plus radiotherapy plus adjuvant chemotherapy, RT only, RT plus adjuvant CT, cCRT only, chemotherapy only or best supportive care (BSC). These groups were also categorized into no treatment (BSC), single modality (radiotherapy, surgery, chemotherapy), double modality (surgery with radiotherapy, or chemotherapy; radiotherapy with chemotherapy) and triple modality (surgery with postoperative radiotherapy and chemotherapy) treatment. Patients that underwent tracheostomy or surgical biopsy alone were classified as best supportive care.

Follow-up was available up to 31 January 2018.

Since data is obtained from the anonymized database from NCR, the ethical board of the METC-LDD provided a waiver from informed consent.

\section{Statistical analysis}

Trends in standardized incidence rates were analyzed using Joinpoint software (version 4.7.0.0). Patient and treatment characteristics were analyzed with descriptive statistics. Overall survival was estimated using the Kaplan-Meier method. Dutch general population data from Statistics Netherlands (CBS) was used for standardized incidences and age-corrected analyses. Different subgroups were compared using the log-rank test. Univariable analyses were done using the variables: sex, age at diagnosis, year of diagnosis, lymph nodes status $(\mathrm{N})$, distant metastases status (M) and treatment. Statistically significant factors were included in a multivariable model. These analyses were performed using the Cox proportional hazards model. Statistical significance was set at a $P$-value of $<0.05$. Statistical program used was SPSS version 23.0.

\section{Results}

A total of 812 patients were identified with ATC over the time period of 1989-2016. Clinical characteristics are shown in Table 1. The age at diagnosis ranged from 29 to 
Table 1 Patient characteristics.

\begin{tabular}{|c|c|c|}
\hline & $n$ & $\%$ \\
\hline Total & 812 & \\
\hline Age (median, range) & 73 (29-99) & \\
\hline \multicolumn{3}{|l|}{ Sex } \\
\hline Male & 237 & 29 \\
\hline Female & 575 & 71 \\
\hline \multicolumn{3}{|l|}{ Year of diagnosis } \\
\hline 1989-1998 & 262 & 32 \\
\hline 1999-2008 & 284 & 35 \\
\hline 2009-2016 & 266 & 33 \\
\hline \multicolumn{3}{|l|}{ N-status } \\
\hline NO & 256 & 32 \\
\hline $\mathrm{N}+$ & 366 & 45 \\
\hline $\mathrm{Nx}$ & 190 & 23 \\
\hline \multicolumn{3}{|l|}{ M-status } \\
\hline MO & 357 & 44 \\
\hline $\mathrm{M}+$ & 342 & 42 \\
\hline $\mathrm{Mx}$ & 113 & 14 \\
\hline \multicolumn{3}{|l|}{ Treatment } \\
\hline No treatment & 295 & 36 \\
\hline Surgery & 96 & 12 \\
\hline Surgery + RT & 124 & 15 \\
\hline Surgery + cCRT & 12 & 2 \\
\hline Surgery + RT + CT & 21 & 3 \\
\hline Surgery + CT & 7 & 1 \\
\hline $\mathrm{RT}$ & 223 & 28 \\
\hline cCRT & 6 & 1 \\
\hline $\mathrm{RT}+\mathrm{CT}$ & 24 & 3 \\
\hline $\mathrm{CT}$ & 4 & 1 \\
\hline \multicolumn{3}{|l|}{ Vital status } \\
\hline Dead & 784 & \\
\hline Alive & 28 & \\
\hline Follow-up in weeks (median, range) & $10(1-1418)$ & \\
\hline
\end{tabular}

cCRT, concurrent chemo-radiotherapy; CT, chemotherapy; RT, radiotherapy.

99 , with a mean of 72 and median of 73 years. There was no significant change in age at diagnosis over the years studied. There were 575 (71\%) female and 237 (29\%) male patients. Mean age significantly differed between male (69 (s.D. 11.2)) and female (74 (s.D. 11.0)) patients $(P<0.001)$. Local disease without regional or distant metastases at diagnosis was found in 151/812 (19\%) patients.

Almost half of the patients $(366 / 812,45 \%)$ had tumor positive cervical lymphadenopathy, of which 197/366 (54\%) patients had bilateral, midline or contralateral metastasis (N1b). Distant metastases at diagnosis were found in 342/812 (42\%) patients. Most of these patients had lung metastases (210/342, 61\%). Sixty-six of 342 (19\%) patients had distant metastases at multiple organ sites.

Active treatment was given in almost two-third of the patients $(517 / 812,64 \%)$. Monotherapy (surgery, radiotherapy or chemotherapy) was given in 323/812 patients $(40 \%)$. Double multimodality treatment
(surgery+RT, cCRT, surgery +CT, RT+adjuvant CT) was given in 161/812 patients (20\%). Triple multimodality treatment (surgery + cCRT, surgery $+\mathrm{RT}+$ adjuvant CT) was given in $33 / 812$ patients $4 \%$ ).

\section{Incidence}

The mean standardized incidence rate (SIR) of ATC over the years was 0.18 per 100000 , ranging from 0.11 to 0.27 per 100 000. There was a significant trend over the years with an estimated annual percentage change (EAPC) of $1.3 \%$ per year (95\% CI: $0.4-2.1 \%)$.

The mean SIR in male patients was 0.10 per 100000 (range 0.05-0.21 per 100 000). There was a significant trend over time with an EAPC of $2.4 \%(95 \% \mathrm{CI}$ : $0.5-4.3 \%)$. For females, the mean SIR was 0.25 per 100000 (range: $0.15-0.40$ per 100 000). There was no significant trend over time with an EAPC of $0.9 \%$ (95\% CI: -0.2 to $1.9 \%)$. Age-adjusted incidence rates showed a EAPC of $3.4 \%$ (95\% CI: -0.1 to $7.0 \%)$.

\section{Overall survival}

The median overall survival time was 2.2 months (95\% CI 1.9-2.5 months). The estimated 1-year survival rate was $12.0 \%$. Patients without distant metastases at diagnosis had an estimated 1-year survival rate of $21.6 \%$. Estimated 2 -and 5-year overall survival was $8.5 \%$ and $5.1 \%$, respectively.

Patients that underwent only best supportive care had a median survival of 3.0 weeks (95\% CI: 2.4-3.7).

Monotherapy $(n=323)$ resulted in a median overall survival of 2.9 months (95\% CI: 2.4-3.4 months) (Fig. 1). There was no statistically significant difference between radiotherapy alone, surgery alone or chemotherapy alone $(P=0.617)$.

Double therapy $(n=155)$ resulted in a median overall survival of 6.9 months (95\% CI: 5.1-8.8 months). The overall survival of the patients in which surgery was part of the treatment appeared slightly better (surgery+RT median 8.0 months (95\% CI: 5.4-10.5) and surgery+CT median 5.5 months (95\% CI: 0.4-10.6)) than the overall survival of the patients that did not undergo surgery (RT + CT median 3.1 months (95\% CI: 2.0-4.3) and cCRT median 2.8 months (95\% CI: 0-9.8)) ( $P=0.009)$ (Fig. 1).

Triple therapy $(n=33)$ resulted in a median overall survival of 6.7 months (95\% CI: 4.2-9.3). Surgery+cCRT patients had a median overall survival of 7.5 months (95\% CI: 3.5-11.4 months) and Surgery+RT+adjuvant CT a median overall survival of 6.5 months 


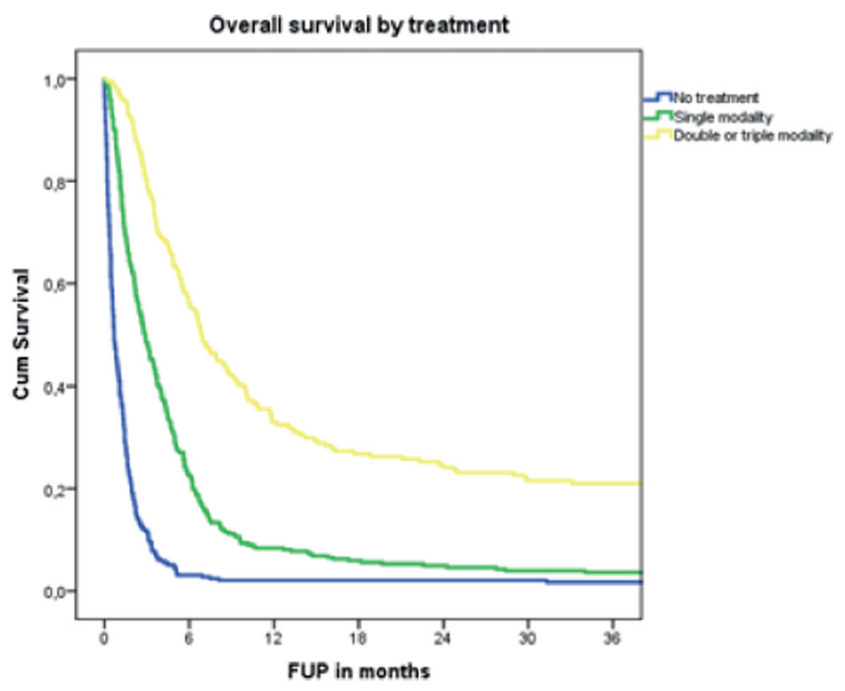

Figure 1

Kaplan-Meier curve for overall survival of anaplastic thyroid cancer patients shown by treatment.

(95\% CI: 4.6-8.4 months). There was no statistically significant difference between the latter two groups.

Patients with stage IVA and stage IVB disease treated with multimodal treatment have equal survival estimates (median 10.0 and 10.5 months/1-year OS $44 \%$ and $45 \%$ ). Multimodal treated patients with stage IVC disease had significantly worse survival estimates (median 3.5 months/1year OS 8\%), compared to stage IVA and stage IVB, $P<0.001$.

Patients surviving $>2$ years had significantly less bilateral lymph node metastases (10\% vs $25 \%$ ), distant metastases ( $8 \%$ vs $45 \%$ ), underwent more often double or triple therapy (69\% vs 19\%) and were younger (age below 65 years $48 \%$ vs 24\% (all with an $P<0.001$ ). In a multivariable Cox regression model, multiple negative prognostic factors were identified: age at diagnosis, year of diagnosis, tumor positive cervical lymph nodes at time of diagnosis and distant metastases at time of diagnosis (Table 2).

\section{Discussion}

This nationwide analysis of ATC patients shows an overview of the incidence and survival over the past decades in The Netherlands. The incidence of about 1.2 per 1000000 in The Netherlands is somewhat in line with the incidences in the United States (4). The most important findings were the increase in incidence of ATC over the years and the increase in incidence of ATC in male patients.

The incidence increase over the last decades has been described by several other studies for thyroid cancer in general $(13,14)$. The incidence increase of ATC specifically

Table 2 Prognostic factors for death.

\begin{tabular}{|c|c|c|c|c|c|c|}
\hline & \multicolumn{3}{|c|}{ Univariable } & \multicolumn{3}{|c|}{ Multivariable } \\
\hline & $\mathrm{HR}$ & $95 \% \mathrm{Cl}$ & $P$-value & $\mathrm{HR}$ & $95 \% \mathrm{Cl}$ & $P$-value \\
\hline Female & Ref & & & & & \\
\hline Male & 0.9 & $0.8-1.1$ & 0.259 & & & \\
\hline Year of diagnosis & 1.007 & 0.999-1.016 & 0.106 & & & \\
\hline Age & 1.028 & $1.022-1.035$ & $<0.001$ & 1.014 & $1.006-1.020$ & $<0.001$ \\
\hline NO & Ref & & & Ref & & \\
\hline $\mathrm{N}+$ & 1.5 & $1.3-1.7$ & $<0.001$ & 1.2 & $1.0-1.4$ & 0.020 \\
\hline $\mathrm{Nx}$ & 1.7 & $1.4-2.1$ & $<0.001$ & 1.2 & $1.0-1.5$ & 0.050 \\
\hline MO & Ref & & & Ref & & \\
\hline M1 & 2.4 & $2.1-2.9$ & $<0.001$ & 1.8 & $1.5-2.1$ & $<0.001$ \\
\hline $\mathrm{Mx}$ & 1.5 & $1.2-1.8$ & 0.001 & 1.2 & $1.0-1.5$ & 0.117 \\
\hline No treatment & Ref & & & Ref & & \\
\hline Surgery & 0.4 & $0.3-0.5$ & $<0.001$ & 0.5 & $0.4-0.6$ & $<0.001$ \\
\hline Surgery + RT & 0.2 & $0.1-0.2$ & $<0.001$ & 0.2 & $0.2-0.3$ & $<0.001$ \\
\hline Surgery + cCRT & 0.1 & $0.1-0.2$ & $<0.001$ & 0.2 & $0.1-0.4$ & $<0.001$ \\
\hline Surgery + RT + CT & 0.2 & $0.1-0.4$ & $<0.001$ & 0.2 & $0.1-0.6$ & 0.001 \\
\hline Surgery + CT & 0.4 & $0.3-0.4$ & $<0.001$ & 0.4 & $0.3-0.5$ & $<0.001$ \\
\hline RT & 0.2 & $0.1-0.5$ & $<0.001$ & 0.3 & $0.1-0.7$ & 0.005 \\
\hline cCRT & 0.2 & $0.1-0.3$ & $<0.001$ & 0.3 & $0.2-0.4$ & $<0.001$ \\
\hline $\mathrm{RT}+\mathrm{CT}$ & 0.3 & $0.2-0.4$ & $<0.001$ & 0.4 & $0.2-0.6$ & $<0.001$ \\
\hline CT & 0.4 & $0.2-1.2$ & 0.107 & 0.6 & $0.2-1.5$ & 0.234 \\
\hline
\end{tabular}

https://eje.bioscientifica.com 
has been described based on SEER data, with an average annual percentage change of 3\% (15). The rising incidence in males specifically has not been described before in the ATC population. Still, thyroid cancers in general are more common in women compared to men. It has been described up to seven times more likely (4). In our study population, there is also a female predominance (71 vs $39 \%)$ in ATC; however, it is less pronounced as in welldifferentiated thyroid cancer.

Main explanations for the rise in incidence of all thyroid cancers are overdiagnosis and changes in environmental factors (like exposure to ionizing irradiation at childhood). The argument of overdiagnosis does not hold for ATC, since the tumor growth is that explosive that it is hard to miss. Accidental finding of an ATC is not that common as it is for well-differentiated thyroid cancer. Environmental factors could play a role in the rising ATC incidence. A major risk factor for the development of ATC is previous benign or malignant thyroid disease $(3,16)$. Despite all, ATC is most commonly seen in elderly patients, which means that age maybe the strongest predictive factor for the development of ATC. So it could be that aging of the general population contributes to the rising incidence found in the present study. Ageadjusted rates in our study are not conclusive, meaning that aging of the population is not explaining the entire rise, but we need to take aging into account interpreting the incidence rise. A more hypothetical explanation for the rise in ATC could be that, over the years, physicians tend to proof every suspected malignancy, even in rapid growing tumors like ATCs. Almost doubling of the histology exams registered in PALGA since 1991 may be an indications for this statement (17).

\section{Survival}

Overall survival in ATC is already long known to be poor (3). Also in our study the overall survival was poor, with an estimated 1-year survival of $12 \%$. A remarkable finding of the study regarding overall survival is the difference between several untreated and treated groups, especially the importance of adjuvant treatment after surgery. Surgery alone showed roughly the same outcome as radiotherapy alone. Albeit, combining surgery with any form of adjuvant treatment seems to increase survival. Despite all treatment, mortality within 1 year is above $90 \%$, but there appeared to be small but substantial group of patients with prolonged survival. Approximately 5-8\% of all the patients do survive longer than 1 year and some significantly longer. Looking more closely to the data of the group of patients (without distant metastases) who underwent surgery $+(\mathrm{C}) \mathrm{RT}$, this percentage of patients surviving over a year rises to $20 \%$ (Fig. 1). So there seems to be a subgroup of patients that do benefit from aggressive therapy. These results are in line with the findings of a Korean study within 19 hospitals (18). They analyzed 348 patients with ATC over a 12-year period and found a median disease specific survival of 15 months and 1 -year survival of $50 \%$ in patients that underwent curative resection and adjuvant RT/cCRT. Another large series from Japan (19), including patients from 38 centers, evaluated 677 patients. They found a long-term (defined as $>1$ year) survival of $15 \%$, of which most patients underwent surgery with any form of adjuvant therapy. They identified the following negative prognostic factors in their patient cohort: extrathyroidal extension, age above 70, leukocytosis, tumors larger than $5 \mathrm{~cm}$ and distant metastases. The single center retrospective study from MD Anderson (2) also points out that tri-modal treatment showed the best overall survival and that development of distant metastases is the main cause for the steep decrease of the survival curve in the first year. The authors of the SEER data analysis $(n=735)$ by Huang et al. (20) showed that (near) total thyroidectomy is the best surgical strategy. Patients with total thyroidectomy had superior OS (HR 0.65) compared to those patients in which total thyroidectomy was not performed. This analysis is biased by the fact that total thyroidectomy is often not possible in case of the biologically more aggressive (bigger and more infiltrative) tumors.

\section{Advantages and limitations}

The advantage of the present study is the nationwide population coverage of the data, which makes reliable incidence and overall survival analyses possible. Also the crosslinked connection between two databases (PALGA and NCR) makes the dataset reliable and robust. The retrospective design, however, is a limitation. Several treatment details are lacking, because this information is not registered by the NCR or PALGA. So details of surgery, radiotherapy and chemotherapy are not available, but absolutely desirable for more solid conclusions.

\section{Future perspectives}

Anaplastic thyroid carcinoma overall has a very dismal prognosis. This study showed that there appears to be 
a subgroup of patients with prolonged survival, but identification of that specific patient group remains hard. Future research needs to focus on the integration of molecular analyses data and clinical patient parameters to help identify the subgroup that may benefit from aggressive treatment. There are some publications on the genomic landscape of ATC $(21,22)$. The study of Kasaian et al. (21) showed the genomic differences between anaplastic and differentiated thyroid cancer which may explain the differences in response to targeted therapy between both groups. The study of Wu et al. (7) showed that BRAF V600E mutations were found in $23 \%$ of the patients and PDL-1 expression was also found in $23 \%$ of the patients. A phase II trial in BRAF V600-mutant anaplastic thyroid cancer patients showed activity of BRAF inhibitors dabrafenib and trametinib (23). However, the genomic signature of ATCs is very heterogeneous. It would be useful to not only focus on targetable mutations but also link genomic signatures to treatment outcomes and survival in order to discriminate patients that survive longer from patients that progress rapidly. Briefly, we want a tool to know upfront which patients may benefit from aggressive therapy and which patients do not. More detailed genomic signature and treatment data of a large nationwide dataset would be of utmost importance to define such a tool.

\section{Conclusion}

In conclusion, ATC is a rare, almost invariably lethal form of thyroid cancer, with a median survival of 2 months and a 1 -year survival of $10 \%$. The incidence is slightly rising in The Netherlands over the past 3 decades. There appears to be a subgroup of patients, mainly those with relatively limited disease and double or triple therapy, that do survive longer and further research is needed to define these patients more distinctively.

\section{Declaration of interest}

The authors declare that there is no conflict of interest that could be perceived as prejudicing the impartiality of this study.

\section{Funding}

This research did not receive any specific grant from any funding agency in the public, commercial or not-for-profit sector.

\section{Acknowledgements}

The authors thank the registration team of the Netherlands Comprehensive Cancer Organisation (IKNL) for the collection of data for The Netherlands Cancer Registry as well as IKNL staff for scientific advice.

\section{References}

1 Kebebew E, Greenspan FS, Clark OH, Woeber KA \& McMillan A. Anaplastic thyroid carcinoma. Treatment outcome and prognostic factors. Cancer 2005103 1330-1335. (https://doi.org/10.1002/ cncr.20936)

2 Rao SN, Zafereo M, Dadu R, Busaidy NL, Hess K, Cote GJ, Williams MD, William WN, Sandulache V, Gross N et al. Patterns of treatment failure in anaplastic thyroid carcinoma. Thyroid $2017 \mathbf{2 7}$ 672-681. (https://doi.org/10.1089/thy.2016.0395)

3 Are C \& Shaha AR. Anaplastic thyroid carcinoma: biology, pathogenesis, prognostic factors, and treatment approaches. Annals of Surgical Oncology 200613 453-464. (https://doi.org/10.1245/ ASO.2006.05.042)

4 Aschebrook-Kilfoy B, Ward MH, Sabra MM \& Devesa SS. Thyroid cancer incidence patterns in the United States by histologic type, 1992-2006. Thyroid 201121 125-134. (https://doi.org/10.1089/ thy.2010.0021)

5 Smallridge RC, Ain KB, Asa SL, Bible KC, Brierley JD, Burman KD, Kebebew E, Lee NY, Nikiforov YE, Rosenthal MS et al. American Thyroid Association guidelines for management of patients with anaplastic thyroid cancer. Thyroid 201222 1104-1139. (https://doi. org/10.1089/thy.2012.0302)

6 Haddad RI, Lydiatt WM, Ball DW, Busaidy NL, Byrd D, Callender G, Dickson P, Duh QY, Ehya H, Haymart M et al. Anaplastic thyroid carcinoma, version 2.2015. Journal of the National Comprehensive Cancer Network 201513 1140-1150. (https://doi.org/10.6004/ jnccn.2015.0139)

$7 \mathrm{Wu}$ H, Sun Y, Ye H, Yang S, Lee SL \& de las Morenas A. Anaplastic thyroid cancer: outcome and the mutation/expression profiles of potential targets. Pathology Oncology Research 201521 695-701. (https://doi.org/10.1007/s12253-014-9876-5)

8 Pezzi TA, Mohamed ASR, Sheu T, Blanchard P, Sandulache VC, Lai SY, Cabanillas ME, Williams MD, Pezzi CM, Lu C et al. Radiation therapy dose is associated with improved survival for unresected anaplastic thyroid carcinoma: outcomes from the National Cancer Data Base. Cancer 2017123 1653-1661. (https://doi.org/10.1002/ cncr.30493)

9 Swaak-Kragten AT, de Wilt JH, Schmitz PI, Bontenbal M \& Levendag PC. Multimodality treatment for anaplastic thyroid carcinoma - treatment outcome in 75 patients. Radiotherapy and Oncology 200992 100-104. (https://doi.org/10.1016/j. radonc.2009.02.016)

10 Wendler J, Kroiss M, Gast K, Kreissl MC, Allelein S, Lichtenauer U, Blaser R, Spitzweg C, Fassnacht M, Schott M et al. Clinical presentation, treatment and outcome of anaplastic thyroid carcinoma: results of a multicenter study in Germany. European Journal of Endocrinology 2016175 521-529. (https://doi.org/10.1530/ EJE-16-0574)

11 van der Sanden GA, Coebergh JW, Schouten LJ, Visser O \& van Leeuwen FE. Cancer incidence in the Netherlands in 1989 and 1990: first results of the nationwide Netherlands cancer registry. Coordinating Committee for Regional Cancer Registries. European Journal of Cancer 1995 31A 1822-1829. (https://doi. org/10.1016/0959-8049(95)00355-m)

12 Fritz A. International Classification of Disease for Oncology. Geneva: World Health Organization, 2000.

13 Lim H, Devesa SS, Sosa JA, Check D \& Kitahara CM. Trends in thyroid cancer incidence and mortality in the United States, 1974-2013. JAMA 2017317 1338-1348. (https://doi.org/10.1001/ jama.2017.2719)

14 Olson E, Wintheiser G, Wolfe KM, Droessler J \& Silberstein PT. Epidemiology of thyroid cancer: a review of the National Cancer Database, 2000-2013. Cureus 201911 e4127. (https://doi. org/10.7759/cureus.4127) 
15 Janz TA, Neskey DM, Nguyen SA \& Lentsch EJ. Is the incidence of anaplastic thyroid cancer increasing: a population based epidemiology study. World Journal of Otorhinolaryngology: Head and Neck Surgery 20195 34-40. (https://doi.org/10.1016/j. wjorl.2018.05.006)

16 Demeter JG, De Jong SA, Lawrence AM \& Paloyan E. Anaplastic thyroid carcinoma: risk factors and outcome. Surgery $1991 \mathbf{1 1 0}$ 956-961; discussion 61-63.

17 https://www.palgaopenbaredatabank.nl/menu/main/dataexplorer? entity=PalgaSample\&hideselect=true. Accessed on 10 July 2019.

18 Baek SK, Lee MC, Hah JH, Ahn SH, Son YI, Rho YS, Chung PS, Lee YS, Koo BS, Jung KY et al. Role of surgery in the management of anaplastic thyroid carcinoma: Korean nationwide multicenter study of 329 patients with anaplastic thyroid carcinoma, 2000 to 2012. Head and Neck 201739 133-139. (https://doi.org/10.1002/hed.24559)

19 Sugitani I, Miyauchi A, Sugino K, Okamoto T, Yoshida A \& Suzuki S. Prognostic factors and treatment outcomes for anaplastic thyroid carcinoma: ATC Research Consortium of Japan cohort study of 677 patients. World Journal of Surgery 201236 1247-1254. (https://doi. org/10.1007/s00268-012-1437-z)
20 Huang NS, Shi X, Lei BW, Wei WJ, Lu ZW, Yu PC, Wang Y, Ji QH $\&$ Wang YL. An update of the appropriate treatment strategies in anaplastic thyroid cancer: a population-based study of 735 patients. International Journal of Endocrinology 20192019 8428547. (https:// doi.org/10.1155/2019/8428547)

21 Kasaian K, Wiseman SM, Walker BA, Schein JE, Zhao Y, Hirst M, Moore RA, Mungall AJ, Marra MA \& Jones SJM. The genomic and transcriptomic landscape of anaplastic thyroid cancer: implications for therapy. BMC Cancer 201515 984. (https://doi.org/10.1186/ s12885-015-1955-9)

22 Weinberger P, Ponny SR, Xu H, Bai S, Smallridge R, Copland J \& Sharma A. Cell cycle M-phase genes are highly upregulated in anaplastic thyroid carcinoma. Thyroid 201727 236-252. (https://doi. org/10.1089/thy.2016.0285)

23 Subbiah V, Kreitman RJ, Wainberg ZA, Cho JY, Schellens JHM, Soria JC, Wen PY, Zielinski C, Cabanillas ME, Urbanowitz G et al. Dabrafenib and trametinib treatment in patients with locally advanced or metastatic BRAF V600-mutant anaplastic thyroid cancer. Journal of Clinical Oncology 201836 7-13. (https://doi.org/10.1200/ JCO.2017.73.6785)

Received 5 February 2020

Revised version received 1 April 2020

Accepted 26 May 2020 